\title{
PREDICTING LIFE SATISFACTION BASED ON SELF-EFFICACY AND SOCIAL SUPPORT
}

\author{
Fahimeh Alipour \\ M.S. in General Psychology, Islamic Azad University of Arak, Arak, Iran \\ Mahta.sm197@,gmail.com \\ Dr. Davoud Taghvaei \\ Assistant Professor And Faculty Member at Islamic Azad University of Arak, Arak, Iran
}

\begin{abstract}
This study aims to predict the correlation between life satisfaction and self-efficacy and social support. The sample consists of 150 female teachers of high school in Qom who were selected by multisectional random sampling. All participants were studied by three tests of Diner life satisfaction, sharer selfefficacy, and Philips social support. The findings showed that there is a significant correlation between life satisfaction, self-efficacy, and social support. The correlation coefficients are respectively 0.361 and $0.469(\mathrm{p}<0.05)$. The results also indicate that life satisfaction can be predicted by subscales of social support, social support by family, friends, and others.
\end{abstract}

Keywords: life satisfaction, self-efficacy, social support

\section{STATEMENT OF PROBLEM}

Happy experience and life satisfaction are the ultimate goal of people's purpose. Therefore, all people are trying to achieve it during their life (Haebner, 2000).

Life satisfaction is one of the oldest and stable isuues in studies related to adults that is considered as general study of the current situation by comparing the person's willings to real access it (Bechtold, 2004).

Ingelehart defines the concept of satisfaction as: life satisfaction reflects the balance between the personal wishes and his current condition. In other word, whatever the gap between the person's wishes and his current condition id high, his satisfaction would be reduced (Ingelhart, 1994 cited in Zaki, 2007).

However life satisfaction depends on personal conditions, the social factors are so effective in increasing and reducing it. The society creates the conditions that can direct the people to achieve the personal wishes and goals, in other hand, it creates a space that the person can interact and communicate with those who provides peace, security, and confidence for an appropriate life. The general life satisfaction is composed of the personal and social conditions and in fact, it is a sign of positive attitudes toward the world and the environment where he lives in.

On of the effective factors on the life satisfaction is self-efficacy. Self-efficacy is one of the important structures on cognitive-social theory by Bandura, and it means the confidence and person's belief toward his abilities in controlling thoughts, senses, activities, and the effective performance in stressful conditions (Caprara, Regalia \& Scabini, 2002). This theory emphasizes on the role of the beliefs of selfefficacy in growth of person's behavior (Meecel, Glienke \& Burg, 2006). Therefore, it affects the real performance of person, selections, organizing and implementing the practices in achieving the performance levels, progress, and the person's effort in an activity (Regalia \& Bandura, 2002). Vecchio, 
Maria, Pastorelli, Del Bove \& Caprara (2007) in a study on the teenagers have found that the academic and social self-efficacy beliefs are as the best predictors of life satisfaction.

Another variables have the close correlation with life satisfaction and social support. The results achieved by Borna Savari (2007) in a study on 190 students indicate that there is positive correlation between social support and life satisfaction of girls and boys. Review of the results of studies show that life satisfaction can be measured significantly and it can be reduced or increased remarkably. In other word, life satisfaction can be increased by identifying the effective factors. Thus, by inspiring the theoretical principle and studies, this study follows to answer this question: is there any correlation between life satisfaction and variables such as self-efficacy and social support?

\section{RESEARCH HYPOTHESIS}

- $\quad$ There is a correlation betweeb self-efficacy and life satisfaction.

- There is a correlation berween social support and life satisfaction.

- There is a correrlation between the subscale of social support by family and life satisfaction.

- $\quad$ There is a correrlation between the subscale of social support by friends and life satisfaction.

- $\quad$ There is a correrlation between the subscale of social support by others and life satisfaction.

\section{THEORIES RELATED TO LIFE SAATISFACTION}

\section{VENTGOT, MERICK, AND ANDESRON'S THEORY}

According to Ventgot and colleagues's theory (2003), the general theory of life satisfaction is a comprehensive theory that includes eight real theories in subjective-objective range. Following, we discuss on life satisfaction in this range based on Ventgot and colleagues' theory:

Subjective life satisfaction means the good sense of person about the life. Everybody can evaluate his view on the objects, senses, and beliefs. Whether the person satisfies his life or not is the aspect that reflects the life quality.

The existing life satisfaction means the person's life in deep level. It is supposed that the person has deeper nature that deserves respect. The person may think some of his biological nees should be met. These factors should be optimized such as growth condition or all should live based on religious ideals that are mixedwith their nature.

Objective life satisfaction means how the person'd life can be d perceived. This view is influenced by the culture that person lives based on that.

The subjective-objective range of life satisfaction is composed of some life satisfaction theories, so this range is called the general theory of life satisfaction. The different issues of the general theory of subjective and objective life quality is based on Ventgot and colleagues' theory (2003) as follow:

Rehabilitation: It is the most natural aspect of lifesatisfaction. Life satisfaction is considered as evaluation of personal life satisfaction.

- $\quad$ Life satisfaction: Life satisfaction means that we feel life ass it flows as it should be. Stisfaction is a subjective status.

- Happy: Sense of happiness does not only mean being happy. It's an especial feeling that is desirable and valuable, but it is achieved hard. Happiness is a deep status feeling in person that includes balance and especial symmetry. Happiness is a sense of life satisfaction.

- Meaning of life: It is an important concept that is used rarely. People speak with their clsed friends about the meaning of life. Those who seek to make life meaningful are confused that all aspects of life seemed different (Eslami, 2005). 
- Biologic view of life satisfaction: this kind of life satisfaction relates to the base of biologic composition in human. In biologic view, human is an alive organism as a set of cells that transfers information because of expansion and provides deep potential for human.

- Understanding life potential: Human uses his potential capacity for constructive activities, good social communications, job and family.

- Meeting the needs: The concept of meeting the needs compared to previous aspects has a lower level of abstraction, while it is more superficial, and it is used in generally. Traditionally needs are related to life satisfaction. This means that when humanitarian needs were fulfilled, life satisfaction increases. Needs are the state of nature, something that all human beings have in common.

- Objective factors: Objective aspects of life satisfaction were related to external factors and detecting them is easy. These factors include income, financial, health, and the number of daily calls with others.

All of these theories are aspects of life that can be placed on-line continuum of mental than physical. Welfare and objective factors are the most superficial aspects because these are related to the surface of our ability to adapt to our culture. Life satisfaction and meet the needs associated with a deeper aspect, instead of happiness and the deepest understanding of the potential of our life and nature that surrounds people. Meaningfulness of life and order and harmony in the most intimate aspects are related to human biological information system. We also need to assume a core of abstract meaning in life, order and harmony in the biological systems that are unspeakable and non-quantifiable, so that the core objective and subjective aspects are the same (Ventegodt, Merrick, and Anderson, 2003).

\section{HIERARCHY OF NEEDS THEORY (MASLOW)}

Maslow (1945; quoted from Eslami, 2005) pyramid of needs stated that the level of physiological needs, safety, belongingness and affection, respect and self-actualization have been combined. His theory helps to understand life satisfaction and life satisfaction by identifying the relevant dimensions and determine the dimensions may be different in different times.

Jean life satisfaction theory

Jean (1992) has provided a model for life satisfaction. According to him, life satisfaction is a multidimensional concept that includes "personal factors", "health or health-related factors", "social, cultural, and environmental factors". According to his model, life satisfaction is influenced by personal grounds, health, socio-economic factors, culture, environment and age. Perceptions of life satisfaction are created as a result of the interaction between man and his environment.

\section{Systematic Theory}

Hagraty (2001, cited in Hemmati, 2007) offered model of life satisfaction based on systems theory approach for public policy implications related to life satisfaction.

\section{The concept of self-efficacy}

Self-efficacy means perceived ability of the individual to adapt the specific situations, and people judge their abilities to carry out a task or adapt a particular situation. Self-efficacy refers to feel self-esteem and self worth in dealing with life (Bandura, 1997). Pour Afkari (2006) knew individual beliefs about the ability to cope with different situations and efficacy.

Efficacy in adolescence depends on early confidence in the ability of a person. In the youth stage efficacy has a major effect on compatibility in marriage, parenting and puts control and domination on the job. In the period between years due to the fact that people constantly assess their abilities, skills, and targets, they are always facing with fresh sources of stress. It is difficult to evaluate the efficacy with age; reduce physical and mental ability, retirement and withdrawal from active work life as a new horizon of selfassessment. Reducing the efficacy of these assessments has a negative effect on mental and physical 
functions, such as sexual activity, physical and intellectual.

In short, the sense of low self-efficacy reduces the gradual loss of cognitive function and behavior and her interests and skills.

Matthews (1988, translation of the nose, 1386) quotes Plato said: "The reality is created by the mind, the life of every person is made of his ideas." The Bible also says: "Man is what has thought about that during a day."

Ellis (1979) knew irrational beliefs cause disproportionate negative emotions such as extreme shyness, guilt, low tolerance for frustration, feelings of being released, extreme perfectionism, anger, resentment and low self-efficacy severe family And to deal with it using rational-emotional therapy techniques recommended to explore the irrational beliefs that aside it, we can coped with problems, increase efficacy and happy life (translation Firouzbakht and Erfani, 2008).

\section{THEORIES AND MODELS OF SOCIAL SUPPORT}

Models and theories of social support have been provided that it can be divided into the following groups. Absence model

In 1989, Gerhard model suggests social factors that this model is result of health effectiveness. This model that is known as absence model suggests people's characteristics that are influenced by missing job, spouse, social class, and friends. Those who are vulnerable are not so supported to overcome the problem of missing.

\section{THE DIRECT IMPACT OF SOCIAL PROTECTION}

The most famous models of social protection is the impact direct. It argues that having social support is beneficial to health, conversely, the absence or lack of social support has a negative impact on health. For example, people who have high social support have greater self-esteem. Such a situation creates a positive attitude regardless of how much stress the individual may have, for example, by making him resistant to infection. Some evidence indicates that higher levels of social protection is to encourage people to adopt more healthy lifestyles (Brown, 1986, quoted by Mardani Rad, 2013). Of course, this is when there is positive support interaction. Otherwise criminal groups' social support leads to a healthy way of life or if this is negative interactions, it has possiblity to predict depression (Schuster, 2000, quoted Hosseini, 1996).

\section{MODEL SHIELDS AND BUMPERS}

The next famous model is the shields or bumpers to reduce stress. It notes that social support protects against the negative effects of too much stress on his health effectively. This protective role is effective only when the person is experiencing severe stress. It protects the cognitive assessment and mitigation through response to the stressful situation is evaluated, it will be done. When a person is faced with a very stressful to those of high social support, position as those who enjoy less protection. For example, a person might offer the perfect solution to his problem or with encouraging words to reassure the person. According to Quatrona and Shor (1990), no matter whether the stress is manageable or not. In the field of controlled events, support information and tools (material) is the most appropriate form of support. Uncontrollable events, emotional support are the best known kind of support. In addition, if the stress of a certain type is the type of needed support, it should be proportional to the stress can affect it.

\section{REVIEW OF LITERATURE}

Khosravi and Nahidpour (2012) in the study of life satisfaction, belief in fairness and social protection in the world and Hindi Iranian students concluded that there is a significant relationship between life satisfaction and social support. 
Hosseini et al (2011) in a study on elderly social support and positive relationship between social support and life satisfaction claimed that social support has a positive effect on physical, psychological, socioeconomic and quality of life and improves the quality of life, makes a good impression on the life and general assessment of life. Kiodo and Abella (2011) considered life satisfaction associated with social support; social interaction and a sense of belonging that is indispensable for the well-being would be increased.

Sarasyno (2010) in a study entitled with social capital and social well-being found that the socialized people in their life are supported socially, and social support leads to a higher life satisfaction and wellbeing. The results of Giolakty (2010) showed that perceived social support predicts life satisfaction 43 percent. He believes that spending time with family has a direct impact on people's satisfaction. He showed that the visuals that are supported by the friends indirectly affect the level of satisfaction and if the person understands himself better than his friends, his satisfaction would be increased. Lent, Taveira, Sheu, and Singley, (2009) showed that the efficacy and environment protection, progress in goals and predict the life satisfaction (Narimani, Eini, Dehqan, Qolamzadeh and Saffarinia, 2013).

\section{METHODOLOGY}

This research is descriptive and correlational. The population of this study consists of all female high school teachers of the course in Qom using multi-stage random sampling from four districts of Qom, regions 1 and 2, and the proportion of teachers in every region of 150 female high school teachers were selected in the second period. 66 people in a school region 1 and 84 schools in region 2 were teaching. Satisfaction with Life Scale, Sherer General Self-efficacy questionnaire and Philips's social support questionnaire were used as the instruments.

\section{SATISFACTION WITH LIFE SCALE (SWLS):}

This scale by Diener, Emmons, Larsen \& Griffin (1985) have been prepared in order to measure life satisfaction. This is a self-report tool that is formed of 5 items and each has 7 options from one (strongly disagree) to seven (strongly agree).

Foreign validity: Diener et al (1985) in a sample of 176 undergraduate students to evaluate their satisfaction with life scale.

Mean and standard deviation of students' scores equal 23.5 and 6.43 respectively and test-retest correlation coefficient score after two months equal 0.82 and alpha coefficient 0.88 . Iranian reliability: in the Iranian et al (2007) standardized the Satisfaction with Life Scale were performed on 109 students.

Cronbach's alpha reliability of this test using 0.83 and test-retest 0.69 are obtained. The reliability obtained 0.89 by Cronbach's alpha in this study.

Foreign validity: life satisfaction scale to determine the validity of which is investigated with a lot of tools. Diener et al. (1985) correlation between Satisfaction with Life Scale scores and other satisfactory means of subjective well-being were reported. In this study, the correlation between life satisfaction and positive and negative affect scales 0.50 and 0.37 are obtained. Schimmack and others (2002) correlation between Satisfaction with Life Scale scores and other satisfactory means of subjective well-being were reported. In this study, the correlation between life satisfaction and positive and negative affect scales 0.46 and 0.48 are obtained. Iranian narrative: The narrative structure of life satisfaction through the use of OHI-oriented traditions and Beck Depression Inventory were calculated. The scale of the OHI positive correlation with the Beck Depression Inventory showed a negative correlation. The research Satisfaction with Life Scale is a useful scale in Iranian psychological research. 
Sherer General Self-efficacy questionnaire

The scale has 17 questions, each question based on the Likert scale ranging from strongly disagree to strongly agree range. This scale has been translated and validated by Bakhtiari Barati (1996).

\section{VALIDITY AND RELIABILITY OF GENERAL SELF-EFFICACY QUESTIONNAIRE}

Bakhtiari Barati (1996) assessed the validity of general self-efficacy scale, scores of this scale was correlated with the size of several character traits

Predictive Self-Efficacy Scale correlation between personality characteristics and size average (6.10, significant at the level 0.05) was confirmed in the direction of the intended structure (quoted Keramati and Shahrarai, 2004). The reliability of scale by using Guttman split-half times 0.76 and 0.79 are obtained by Cronbach's alpha coefficient (Shamaeizadeh and Abedi, 2005). In Vaqari's research (2000) in order to the final analysis of self-efficacy, Cronbach's alpha was obtained 0.85 .

\section{PHILLIPS'S SOCIAL SUPPORT QUESTIONNAIRE}

This questionnaire has been prepared by Wax, Phillips, Helly, Tohmson, Willams and Stwart (1986, quoted by Murad born 2006). The questionnaire has 23 family articles that three of them relate to family (8 $\quad$ items), friends $(7 \quad$ items $)$ and others $(8$ items). The questionnaire for the first time was used by Ebrahim Qavam (1992). He conducted on 100 students and 200 Iranian students and reliability and validity of the instruments. Reliability of the total scale of students at 0.90 and 0.70 were achieved. The test-retest reliability of the questionnaire was 0.81 after six weeks. In another study, Cronbach's alpha for the whole questionnaire was 0.81 (Khoshkonesh et al., 2010). The reliability obtained by Cronbach's alpha in this study is 0.70 . Analyzing

First hypothesis: There is a relationship between self-efficacy and life satisfaction.

Table 1. Pearson correlation test efficacy and life satisfaction $(n=150)$

\begin{tabular}{|l|l|l|l}
\hline Sig. & Effect coefficient & Correlation coefficient & Variable \\
\hline 0.001 & 0.13 & 0.361 & Self-efficacy \\
\hline
\end{tabular}

As can be seen in the table, the correlation between self-efficacy and life satisfaction is equal to 0.361 . This positive correlation is statistically significant $(\mathrm{p}<0.05)$; as a result, the first hypothesis is confirmed. Therefore, we can say that there is a significant relationship between self-efficacy and life satisfaction.

The second hypothesis: There is a relationship between social support and life satisfaction.

Table 2. Pearson correlation test social support and life satisfaction $(n=150)$

\begin{tabular}{|l|l|l|l}
\hline Sig. & Effect coefficient & Correlation coefficient & Variable \\
\hline 0.001 & 0.22 & 0.469 & Social support \\
\hline
\end{tabular}

As it can be seen in the table, the correlation between social support and life satisfaction is equal to 0.469 . This positive correlation is statistically significant $(\mathrm{p}<0.05)$; as a result, the hypothesis is confirmed.

The third hypothesis: -There is a correrlation between the subscale of social support by family and life satisfaction. 
Table 3. Pearson correlation test of social support and life satisfaction $(n=150)$

\begin{tabular}{|l|l|l|l}
\hline Sig. & Effect coefficient & Correlation coefficient & Variable \\
\hline 0.001 & 0.28 & 0.529 & Social support by family \\
\hline
\end{tabular}

As it can be seen in the table, the correlation between social support and life satisfaction is equal to 0.529 . This positive correlation is statistically significant $(\mathrm{p}<0.05)$; as a result, the hypothesis is confirmed. Therefore, we can say that there is a correlation between social support and life satisfaction there.

The fourth hypothesis: there is a correlation between the subscales of social support of friends and life satisfaction.

Table 4. Pearson correlation test social support of friends and life satisfaction $(n=150)$

\begin{tabular}{|l|l|l|l}
\hline Sig. & Effect coefficient & Correlation coefficient & Variable \\
\hline 0.014 & 0.04 & 0.201 & Social support by friends \\
\hline
\end{tabular}

As can be seen in the table, the correlation between social support of friends and life satisfaction is equal to 0.201 . This positive correlation is statistically significant $(\mathrm{p}<0.05)$; as a result, the hypothesis is confirmed. Therefore, we can say that there is a correlation between social support and life satisfaction are friends.

Fifth hypothesis: there is a correlation between the subscales of social support and life satisfaction.

Table 5. Pearson correlation test social support for people with life satisfaction $(n=150)$

\begin{tabular}{|l|l|l|l}
\hline Sig. & Effect coefficient & Correlation coefficient & Variable \\
\hline 0.001 & 0.115 & 0.339 & Social support by others \\
\hline
\end{tabular}

As it can be seen in the table, correlation between social support by others and life satisfaction is equal to 0.339 . This positive correlation is statistically significant $(\mathrm{p}<0.05)$; as a result, the hypothesis is confirmed. Therefore, we can say that there is a correlation between social support by others and life satisfaction.

Sixth hypothesis: there is correlation between self-efficacy, social support and life satisfaction.

Table 6: Results of regression analysis to examine the relationship between multiple input method for self-efficacy and social support and life satisfaction

\begin{tabular}{|c|c|c|c|c|c|c|c|c|c|c|}
\hline Sig. & $\mathrm{T}$ & $\beta$ & $\mathrm{SE}_{\mathrm{b}}$ & B & Sig. & $\mathrm{F}$ & Sig. & $\mathrm{R}^{2}$ & $\mathrm{R}$ & Variable \\
\hline 0.001 & 4.431 & & 4.608 & 20.002 & \multirow[t]{3}{*}{0.001} & \multirow[t]{3}{*}{20.065} & \multirow[t]{3}{*}{0.001} & \multirow[t]{3}{*}{0.356} & \multirow[t]{3}{*}{0.597} & Index \\
\hline 0.001 & 3.287 & 0.228 & 0.063 & 0.208 & & & & & & constant number \\
\hline 0.002 & 3.189 & 0.257 & 0.157 & 0.501 & & & & & & Efficacy \\
\hline
\end{tabular}


As the multiple regression analysis method shows, multiple correlation results show that the correlation between life satisfaction, self-efficacy and social support on the one hand and on the other hand is equal to 0.597 . R 2 equal to 0.356 and show that nearly $6.35 \%$ of the variance in life satisfaction scores are predicted by these variables. The results of analysis of individual variables have shown that among these variables, self-efficacy $(B=0.208, p=0.001)$, social support $(B=0.501, p=0.001)$ with the variable relationship are meaningful. In other words, self-efficacy and social support can predict life satisfaction. With the addition of a single-efficacy and social support, respectively at $0.228,0.311$ and 0.257 is added to the value of life satisfaction. Stepwise multiple regression analysis are presented in Tables 7 and 8 .

Table 7. The multiple correlation coefficient and analysis of variance to predict life satisfaction regarding the efficacy and social support

\begin{tabular}{|l|l|l|l|l|l|l|l}
\hline Sig. & F & Sig. & $\begin{array}{l}\text { F } \\
\text { change }\end{array}$ & $R^{2}$ & $R$ & \multicolumn{2}{|c}{ Variables } \\
\hline 0.001 & 33.117 & 0.001 & 12.939 & 0.311 & $557 / 0$ & Efficacy & 2 \\
\hline 0.001 & 26.61 & 0.001 & 9.683 & 0.353 & $595 / 0$ & social support & 3 \\
\hline
\end{tabular}

The results of stepwise regression analysis showed that self-efficacy and social support predict life satisfaction. With the addition of self-efficacy, predictive power equals 0.311 percent. The two factors predict life satisfaction 31.1. With the addition of social support variables ablity to predict is 0.353 close to $3.35 \%$ of the three variables to predict life satisfaction.

Table 8. Stepwise multiple regression to predict life satisfaction regarding the efficacy and social support

\begin{tabular}{|l|l|l|l|l|l|l|}
\hline Sig. & $\mathrm{T}$ & $\beta$ & $\mathrm{SE}_{\mathrm{b}}$ & $\mathrm{B}$ & \multicolumn{2}{|c}{ Variables } \\
\hline 0.001 & 5.245 & & 4.124 & 21.629 & Index & \\
\hline 0.001 & -3.259 & 0.226 & 0.063 & 0.206 & Efficacy & \\
\hline 0.001 & 3.112 & 0.247 & 0.155 & 0.438 & social support & \\
\hline
\end{tabular}

Based on the results obtained in the above table, the coorelation between self-efficacy and social support and life satisfaction is positive. In other words, with the addition of a single self-efficacy and social support, respectively, to $0.226,0.247$, life satisfaction would be increased.

\section{DISCUSSION AND CONCLUSION}

As seen in the results, there is a significant positive correlation between self-efficacy and life satisfaction, including research done in this area that are consistent with the findings of this study can be noted in the following studies:

Bakoxi and Parker (2006) demonstrated that social self-efficacy and life satisfaction correlate with the academic success, social competence and avoiding risk behaviors.

Wake Chiu, Maria, Bastorly, Dell and Caprara (2007) found that educational and social self-efficacy beliefs are as the best predictors of life satisfaction. Lent, Taveira, Sheu, and Singley, (2009) showed that the efficacy and environment protection, progress in goals and predict the life satisfaction (Narimani, Eini, Dehqan, Qolamzadeh and Saffarinia, 2013). The results showed a significant positive relationship between social support and life satisfaction. In this regard, we can mention the following studies: 
The findings Oates et al (2006) suggest that by increasing the ability of psychological and social support, stress would be decreased and mental health quality of life is good. Asgari, Naderi, and Sharaf al-Din (2009) showed a significant relationship between social anxiety and social support and subjective wellbeing in women.

Kyiodo and Abella (2011) stated that life satisfaction is associated with social support and they believed that social interaction can increase a sense of belonging that is indispensable for the well-being.

According to research findings, significant positive correlation between social support and life satisfaction are the result of family ties. The findings are consistent with findings Hesam and colleagues (2011) in research on the relationship between perceived social support, mental health and life satisfaction in students of Gorgan State University concluded that perceived social support from family predict the changes in life satisfaction $42 \%$. The findings are also consistent with the results of Giolakty (2010) that the perceived social support predicts life satisfaction $43 \%$ and is consistent with spending time in family direct impact on people's satisfaction.

According to the results of this research, there is a significant positive correlation between social support from friends and life satisfaction that is consistent with the following research: Yamavka (2008) evaluated the health and psychological well-being as social capital in East Asia and concluded that there is a positive relationship between psychological well-being and social capital. The people in their lives are more social and more friends are of higher mental well-being as well. The results of Giolakty (2010) showed that the visuals that are supported by the friends indirectly affect the level of satisfaction and if the person understands himself better than his friends, his satisfaction would be increased.

The findings of this study suggest that there is a significant positive correlation between life satisfaction and social support from others. In this hypothesis, there are no studies that have examined the relationship between these two variables. In this context, Lent and colleagues's study (2009) noted that research among students demonstrated efficacy and environmental support to predict life satisfaction. The results also suggest that there is a significant positive correlation between self-efficacy and social support and life satisfaction which shows that the higher the level of self-efficacy and social support, and life satisfaction are also higher.

\section{REFERENCES}

Hossam, Moslem; Asayesh, Hamid; Qorbani, Mostafa; Shariati, Ali Reza; Nasir, Hossein (2011). The relationship between perceived social support, mental health and life satisfaction among students of state universities Gorgan scarifying. Journal of Nursing and Midwifery, Gorgan. Volume VIII. 41-34 Page No. 1.90. Spring and summer.

Hosseini, Ahmad (2011). The effect of social support on social health of women heads of household. Social Research Quarterly, Issue seventh, the summer of 1389. Khosravi, Zohreh, Nahidpour, Farzaneh (2012). Life satisfaction survey, believe in the fairness of world social support pages, Iranian and Indian Journal of Sociology women, Issue II. Asgari, Parviz; Naderi, Farah; Sharaf al-Din, Hoda (2009). The relationship between social anxiety, hope and social support and subjective well-being in females. Woman Magazine and culture, the first, second, winter 2009, Ahvaz Islamic Azad University, 58.

Mardani Rad, Mojgan (2013). Evaluation models of social protection in order to develop an integrated model to explain mental health and quality of life and life satisfaction. PhD thesis, University of Payam Noor. 
Caprara, G. V., Regalia, C., \& Scabini, E. (2002). Autoefficacia familiar (Familiar selfefficacy). In La Valutazione ell'Autoefficacia (Self- Efficacy Assessment) (ed. G.V. Caprara), pp. 63-86. Edizioni Erickson: Trento.

Gülaçti, F. (2010). The Effect of Perceived Social Support on Subjective Well-being, Procedia Social and Behavioral Sciences, 2, 3844-3849.

Lent, W Taveira, M, Sheu, H \& Singley, D, (2009). Social cognitive predictors of academic adjustment and life satisfaction in Portuguese college students: A longitudinal analysis, Journal of Vocational Behavior, 74(2). 190-198.

Quevedo, R.J.M., \& Abella, M.C. (2011). Well-being and Personality: Facet level Analyses, Personality and Individual Differences, 50, 206-211.

Regalia, C. \& Bandura, A. (2002). Longitudinal impact of perceived selfregulatory efficacy on violent conduct. European Psychologist, 7, 63- 69.

Sarracino, F. (2010). Social capital and subjective well-being trends:Comparing 11 western European countries. The Journal of Socio- Economics, Elsevier, 39(4), 482-517.

Ventegodt, S., Merrick, J., \& Anderson, N. J (2003), "Qol I. the I QOL theory: an integrative theory of the global quality of life concept", The scientific world journal, Vol. 3, pp: 1030- 1040.

Yamaoka, K. (2008). Social capital and health and well-being in East Asia: a population-based study. Journal Social Science \& Medicine. 66(4), 885-99.

Vecchio, G, M, Maria, M, Pastorelli, C Del Bove, G \& Caprara, G, V, (2007). Multi-faceted self-efficacy beliefs as predictors of life satisfaction in late adolescence, Personality and Individual Differences, 43(7). $1807-1818$ 
The Turkish Online Journal of Design, Art and Communication - TOJDAC August 2016 Special Edition

Submit Date: 21.06.2016, Acceptance Date: 26.07.2016, DOI NO: 10.7456/1060AGSE/032 Copyright (C) The Turkish Online Journal of Design, Art and Communication 